\title{
Complications of Polycystic Kidney Disease and Relation With Disease Progression: An Observational Retrospective Study
}

Mònica Pérez-Mir ( $\square$ mperezmir@yahoo.com )

Nephrology Department, H. U. Germans Trias i Pujol. Badalona. Spain.

Laura Carreras-Planella

Institut d'Investigació Germans Trias i Pujol. Badalona. Spain.

Francesc Borràs

REMAR-IVECAT group. Germans Trias i Pujol. Health Science Research Institute, Can Ruti Campus, Badalona, Spain.

Josep Bonet

REMAR-IVECAT group. Germans Trias i Pujol. Health Science Research Institute, Can Ruti Campus, Badalona, Spain.

Maribel Troya

Nephrology Department, H. U. Germans Trias i Pujol. Badalona. Spain.

\section{Research Article}

Keywords: autosomal dominant polycystic kidney disease, chronic kidney disease, kidney complications, high blood pressure

Posted Date: December 28th, 2020

DOl: https://doi.org/10.21203/rs.3.rs-122413/v1

License: (a) (1) This work is licensed under a Creative Commons Attribution 4.0 International License. Read Full License 
COMPLICATIONS OF POLYCYSTIC KIDNEY DISEASE AND RELATION WITH DISEASE PROGRESSION: AN OBSERVATIONAL RETROSPECTIVE STUDY

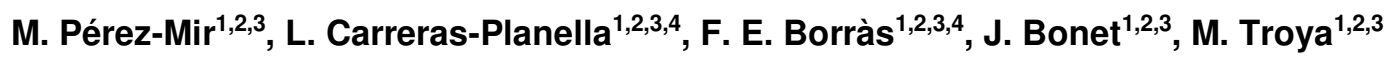

1. Nephrology Department, H. U. Germans Trias i Pujol. Badalona. Spain.

2. Universitat Autònoma de Barcelona, Bellaterra, Cerdanyola del Vallès, Spain.

3. REMAR-IVECAT group. Germans Trias i Pujol. Health Science Research Institute, Can Ruti Campus, Badalona, Spain.

4. Institut d’Investigació Germans Trias i Pujol. Badalona. Spain.

Corresponding author:

Mònica Pérez Mir.

Nephrology Department. H. U. Germans Trias i Pujol.

Ctra. Canyet s/n, 08916, Badalona. Barcelona. Spain.

mperezmir@yahoo.com

0034934978898 


\section{Abstract:}

Background: Autosomal dominant polycystic kidney disease (ADPKD) is a renal inherited disease characterized by the growth of bilateral renal cysts that lead to deterioration in renal function and end-stage renal disease (ESRD). These patients frequently present complications like urinary tract infection, acute pyelonephritis, acute or chronic pain, renal lithiasis or high blood pressure (HBP). The aim of this study is to compare the renal evolution in ADPKD patients with renal complications and/or HBP compared to those without complications nor HBP.

Methods: Observational retrospective study of 29 ADPKD patients with normal renal function and $<70$ years followed up in our center. Clinical and analytical information of 2010, 2015 and 2017 were determined.

Results: 29 patients were enrolled with a median age of 41 years [34-54], eighteen women $(62,1 \%)$ and eleven men. Median of estimation of glomerular function rate (eGFR) was $85.8 \mathrm{ml} / \mathrm{min}$ [70.7-115.6] in 2010, $76.0 \mathrm{ml} / \mathrm{min}$ [57.0-99.9] in 2015 and $63.1 \mathrm{ml} / \mathrm{min}$ [45.0-95.8] in 2017. Eight patients $(27.6 \%)$ have never had kidney complication nor HBP. Nine patients $(31.0 \%)$ have normal blood pressure, 6 others (20.7\%) have well controlled high blood pressure (HBP) and 14 (48.3\%) have badly controlled HBP. When patients were divided between those who have never presented a complication (C-) and those who present renal complication and/or HBP (C+), the first group presented better kidney function. When patients were segregated into those who have never presented complication or well-controlled HBP (CHBP-) and those with renal complication and/or badly-controlled HBP $(\mathrm{CHBP}+)$ no differences were found at the initial eGFR, but a faster worsening of kidney function in $\mathrm{CHBP}+$ group.

Conclusions: In ADPKD patients, the decrease in eGFR is significantly important in individuals showing complications (including HBP) compared to those who did not present complications. Individuals with complications and badly-controlled HBP show even greater differences in kidney function decrease compared with patients without complications or well controlled HBP.

Key words: autosomal dominant polycystic kidney disease, chronic kidney disease, kidney complications, high blood pressure. 


\section{Background:}

Adult autosomal dominant polycystic kidney disease (ADPKD) is the most common renal inherited disease. It is characterized by the growth of bilateral renal cysts that generate very high renal volumes and cause peri-cystic fibrosis. These alterations lead to deterioration in renal function, which can cause end-stage renal disease (ESRD) between the fourth and seventh decades of life [1].

Cystic growth is associated with several renal complications related to higher morbidity and mortality of these patients, with a corresponding increase in hospital admissions and health expense, a challenge that nephrologist face daily. Furthermore, these complications are associated with a rapid progression of kidney deterioration and an early need for renal replacement therapy.

The main kidney complications associated with ADPKD are urinary tract infection (including kidney cyst infections or acute pyelonephritis) [2-3], acute or chronic pain (secondary to hemorrhage or rupture of cysts or the increase in size of the kidneys or liver), or kidney lithiasis.

Also, hypertension is a very frequent manifestation, occurring in $60 \%$ of patients before the decline of the renal function [4]. Hypertension is associated to a higher total kidney volume [5], which is a prognostic biomarker on renal function decline and progression to end-stage renal disease [6].

The prognosis of ADPKD is clearly marked by the progressive deterioration of renal function. This remains stable during the first years despite the growth of renal volume, but after the onset of chronic kidney disease (CKD), the glomerular filtration rate decreases at an average speed of 4.4-5.9ml/min/year [7], although the progression of CKD in ADPKD is very variable; in some cases renal replacement therapy (RRT) may be required before 40 years, while in others it may persist without the need for RRT throughout life. Several factors have been established that can determine the rate of progression to chronic kidney disease, mainly the genetic background (including affected gene and type of mutation) [8-9], total kidney volume or height adjusted total kidney volume [8-10] and high blood pressure [11]. Other possible related factors are early age 
of onset of symptoms and complications, proteinuria, left ventricular hypertrophy, inability to concentrate urine, and low high density lipoprotein (HDL) levels [12].

Progression to end-stage renal disease is highly variable among ADPKD patients, and thus predicting models are difficult to apply on an individual patient basis. Even so, two major models for predicting progression in ADPKD are currently used. The "Mayo model" is based on heightadjusted total kidney volume (hTKV) (measured by TC or MRI image) and age. This method segregates patients into 5 groups (from $1 \mathrm{~A}$ to $1 \mathrm{E}$ ), being group $1 \mathrm{C}$ to $1 \mathrm{E}$ patients at greatest risk of rapid disease progression. The "PROPKD Score" is based on clinical and genetic parameters. This score evaluates gender (male sex, 1 point), hypertension before 35 years (2 points), first urological event before 35 years (cystic infection, haematuria or pain, 2 points) and type and gene of mutation (PKD2 0 points, non-truncating mutation of PKD1 2 points, and truncated mutation of PKD1 4 points) [13-14]. Higher scores imply elevated risk of progression.

Recently approved by the European Medicines Agency (EMA), vasopressin V2 receptor antagonist is a new treatment of choice for these patients. To date, vasopressin V2 receptor antagonist is the first approved drug shown to directly affect disease progression [15]. However, due to some notable side effects associated to the treatment and its high cost, a good stratification is recommendable to prioritise treatment to those patients at higher risk [16].

In this context, only a few studies have compared the rate of renal function deterioration (measured as decrease in eGFR) between patients with and without complications associated to ADPKD. Thus, we aimed to evaluate the number of ADPKD-related complications in our patient cohort in order to ascertain whether these complications lead to a worse evolution of renal function.

\section{Material and methods:}

We performed an observational retrospective study in ADPKD patients aged 18 - 70 with normal renal function.

Study population: Participants were recruited from a wide cohort of patients that are routinely followed up in our center. The diagnosis of ADPKD was made based on Pei's ultrasound criteria 
and previous family history [17]. Patients included were aged $>18$ years and under active followup between 2010 and 2017. The exclusion criteria were: kidney transplant, dialysis, loss of followup and age $>70$ years, as hypertension of these patients would not have a similar etiopathogenic mechanism as hypertension in patients with ADPKD.

All patients provided informed consent and the protocol was conducted following the Helsinki declaration. This study was approved by the Ethical Comité of Hospital Universitari Germans Trias I Pujol, Badalona.

Definitions:

- Arterial hypertension or high blood pressure (HBP) is a long-term medical condition in which the blood pressure in the arteries is persistently elevated $(>140 / 90 \mathrm{mmHg})$. We considered well-controlled HBP patients as those who received antihypertensive drugs and presented normal blood pressure in the doctor's office. We considered badly controlled HBP in those patients with $>140 / 90 \mathrm{mmHg}$ despite antihypertensive drugs or patients with increasing or initiating treatment during the follow-up.

- Kidney complications: we considered patients with kidney complications as those who presented at least one episode of urinary tract infection or acute pyelonephritis, acute or chronic pain and/or renal lithiasis.

- Chronic kidney disease: gradual decrease in kidney function over a period of months or years, characterized by eGFR $<90 \mathrm{ml} / \mathrm{min}$.

Data collection: The visits were carried out at the Hospital Universitari Germans Trias i Pujol (Badalona) by the Nephrology unit staff and followed standard clinical practice.

We performed an observational retrospective study identifying the number of infectious complications (cystic crisis, urinary infection, kidney lithiasis or acute pyelonephritis) and the presence of hypertension for the last 8 years (2010-2017), as well as the analytical results of these years. 
Statistical Methodology: For the statistical analysis we employed the SPSS program. Baseline characteristics are given as medium and standard deviation. For the comparison between groups, we used the Student's T for independent samples.

\section{Results:}

Baseline characteristics: Our centre routinely monitored up to 141 patients for ADPKD during the entire study. The baseline characteristic is summed up in Table 1. Patients that required a renal replacement therapy (73 renal transplant patients, $51.8 \%$, plus 19 patients on dialysis, $13.5 \%$ ) were excluded. In addition, patients with discontinued follow-up (only completing one of the three analysis required in the study, 14 patients, 9.9\%) were also not considered. In addition, patients older than 70 years (5 subjects, 3.6\%) and one other (0.7\%) under 18 years old at recruitment were not included. Thus, a homogeneous sample of 29 patients was finally followed up for the entire period.

Data collection started in September 2017. Patients' median age was 41.0 (34.0-54.5) years, with a proportion of $62,1 \%$ women $(n=18)$. At the initial time point, fifteen patients $(51.7 \%)$ presented chronic kidney disease, as indicated by a reduced eGFR (eGFR $<90 \mathrm{ml} / \mathrm{min} / 1.73 \mathrm{~m}^{2}$ ). The medians of eGFR were $83.7(67.9-111.2) \mathrm{ml} / \mathrm{min} / 1.73 \mathrm{~m}^{2}$ in $2010,76.0(54.9-95.9) \mathrm{ml} / \mathrm{min} / 1.73 \mathrm{~m}^{2}$ in 2015 and $56.5(42.7-92.3) \mathrm{ml} / \mathrm{min} / 1.73 \mathrm{~m}^{2}$ in 2017.

Only 9 patients never presented HBP (31.0\%), whereas 6 have well-controlled hypertension $(20.7 \%)$ and 14 have uncontrolled hypertension (48.3\%) that required initiating or increasing the treatment. 15 patients (51.7\%) have never presented kidney complication. The median of kidney complications of the patients that have had at least one kidney complication was $1.3(0-1.5)$. Only $4(13.8 \%)$ patients have presented neither kidney complication nor HBP. 
Table 1. Baseline characteristics of all 29 patients.

\begin{tabular}{|c|c|}
\hline \multicolumn{2}{|c|}{ Baseline characteristics } \\
\hline Age, years (median (p25-p75)) & $41.0(34.0-54.5)$ \\
\hline Gender, female & $62.1 \%(18)$ \\
\hline 2010 eGFR, $\mathrm{ml} / \mathrm{min} / 1.73 \mathrm{~m}^{2}$ (median (p25-p75)) & $85.8(70.7-115.6)$ \\
\hline 2015 eGFR, $\mathrm{ml} / \mathrm{min} / 1.73 \mathrm{~m}^{2}$ (median (p25-p75)) & $76.0(57.0-99.9)$ \\
\hline 2017 eGFR, $\mathrm{ml} / \mathrm{min} / 1.73 \mathrm{~m}^{2}$ (median (p25-p75)) & $63.1(45.0-95.8)$ \\
\hline $\begin{array}{l}\text { Hypertension } \\
\text { - No HBP } \\
\text { - Controlled HBP } \\
\text { - Uncontrolled HBP }\end{array}$ & $\begin{array}{l}9 \text { patients }(31.0 \%) \\
6 \text { patients }(20.7 \%) \\
14 \text { patients }(48.3 \%)\end{array}$ \\
\hline $\begin{array}{l}\text { Complications: } \\
\text { - Patients without kidney complication } \\
\text { - Patients without complication neither HBP }\end{array}$ & $\begin{array}{l}8 \text { patients }(27.6 \%) \\
4 \text { patients }(13.8 \%)\end{array}$ \\
\hline
\end{tabular}

Median (p25-p75), n (\%), eGFR: estimated glomerular filtration rate. HBP: high blood pressure.

\section{Differences between patients who have presented at least one complication:}

We divided patients into two groups: C- (patients without any complication or HBP) and C+ (patients with at least one kidney complication and/or HBP). Significant differences were found between the two groups, and patients with complication have a worse eGFR in all three analytical controls (Table 2). Also, older patients were significantly over-represented in $\mathrm{C}+$ group. The Cgroup is significantly younger ( 21 years [16.8-47.8] compared to the $C+43$ years [37.5-54.5] with $p=0.05$ ), a possible reason of the increased eGFR in group $C$-. Interestingly, whilst $C$ - patients barely reduced their eGFR by $5 \%$ in 7 years (96.4\% of eGFR at 2017 respect 2010), C+ patients progressively reduced their eGFR by $8,6 \%$ (from 2010 to 2015 ) and by an additional $25 \%$ at later stage (from 2015 to 2017). This eGFR differences are shown in Figure 1, part (1). 
Table 2. Differences between patients with and without complications (including hypertension).

\begin{tabular}{|c|c|c|c|}
\hline & C - & $\mathrm{C}+$ & $\mathbf{p}$ \\
\hline $\mathrm{N}$ & 4 & 25 & \\
\hline Age, years (median (p25-p75)) & $21.0(16.8-47.8)$ & $43.0(37.5-54.5)$ & 0.05 \\
\hline Female $(n, \%)$ & $3(75.0 \%)$ & $16(64.0 \%)$ & $0.67 \dagger$ \\
\hline $\begin{array}{l}2010 \text { eGFR, } \mathrm{ml} / \mathrm{min} / 1.73 \mathrm{~m}^{2} \text { (median (p25- } \\
\mathrm{p} 75) \text { ) }\end{array}$ & $140.3(105.1-169.3)$ & $81.5(68.3-96.8)$ & 0.008 \\
\hline $\begin{array}{l}2015 \text { eGFR, } \mathrm{ml} / \mathrm{min} / 1.73 \mathrm{~m}^{2} \text { (median (p25- } \\
\mathrm{p} 75) \text { ) }\end{array}$ & $133.0(101.6-147.0)$ & $74.5(56.7-94.4)$ & 0.003 \\
\hline $\begin{array}{l}2017 \text { eGFR, } \mathrm{ml} / \mathrm{min} / 1.73 \mathrm{~m}^{2} \text { (median (p25- } \\
\mathrm{p} 75) \text { ) }\end{array}$ & $135.0(98.3-144.0)$ & $56.0(43.5-87.7)$ & 0.004 \\
\hline
\end{tabular}

Median (p25-p75), n (\%), Mann-Whitney test, + x-square test. C-: patients without kidney complication or HBP. C+: patients with kidney complication and/or HBP. eGFR: estimated glomerular filtration rate. HBP: high blood pressure.

Differences between patients showing different control of HBP:

Since well-controlled hypertension causes less organ damage, we made a second division considering in the CHBP- group the patients who have never presented any kidney complication and have not presented HBP or have well controlled HBP, and in the group $\mathrm{CHBP}+$ patients with at least one kidney complication and/or badly controlled HBP. Considering these groups, again we observed that C- patients barely reduced their eGFR by $7 \%$ in 7 years $(92.9 \%$ of preserved eGFR at 2017 respect 2010), whilst C+ patients progressively reduced their eGFR by $16 \%$ (from 2010 to 2015 ) and by an additional $21.2 \%$ at later stage (from 2015 to 2017) (Table 3). This eGFR differences are shown in Figure 1, part (2). 
Table 3. Differences between patients with and without complications (including only badly controlled hypertension).

\begin{tabular}{|c|c|c|c|}
\hline & CHBP - & CHBP + & $\mathbf{p}$ \\
\hline $\mathrm{N}$ & 9 & 20 & \\
\hline Age, years (median (p25-p75)) & $40.0(21.0-56.5)$ & $42.0(35.0-52.5)$ & 0.5 \\
\hline Female (n, \%) & $6(66.7 \%)$ & $13(65.0 \%)$ & $0.64 \dagger$ \\
\hline $\begin{array}{l}2010 \text { eGFR, } \mathrm{ml} / \mathrm{min} / 1.73 \mathrm{~m}^{2} \text { (median (p25- } \\
\mathrm{p} 75) \text { ) }\end{array}$ & $102.0(76.3-141.5)$ & $83.0(67.9-97.4)$ & 0.17 \\
\hline $\begin{array}{l}2015 \text { eGFR, } \mathrm{ml} / \mathrm{min} / 1.73 \mathrm{~m}^{2} \text { (median (p25- } \\
\text { p75)) }\end{array}$ & $94.8(81.7-133.0)$ & $69.8(50.9-89.1)$ & 0.007 \\
\hline $\begin{array}{l}2017 \text { eGFR, } \mathrm{ml} / \mathrm{min} / 1.73 \mathrm{~m}^{2} \text { (median (p25- } \\
\text { p75)) }\end{array}$ & $94.8(53.5-128.4)$ & $55.0(32.2-81.9)$ & 0.038 \\
\hline
\end{tabular}

Median (p25-p75), $n$ (\%), Mann-Whitney test, $+x$-square test. CHBP-: patients with stable blood pressure and without kidney complication. $\mathrm{CHBP}+$ : patients with kidney complication, new diagnosis of HBP and/or badly controlled HBP. eGFR: estimated glomerular filtration rate. HBP: high blood pressure.

Figure 1. Evolution of decrease in eGFR in groups $\mathrm{C}-$ and $\mathrm{C}+(1)$ and $\mathrm{CHBP}-$ and $\mathrm{CHBP}+(2)$ :
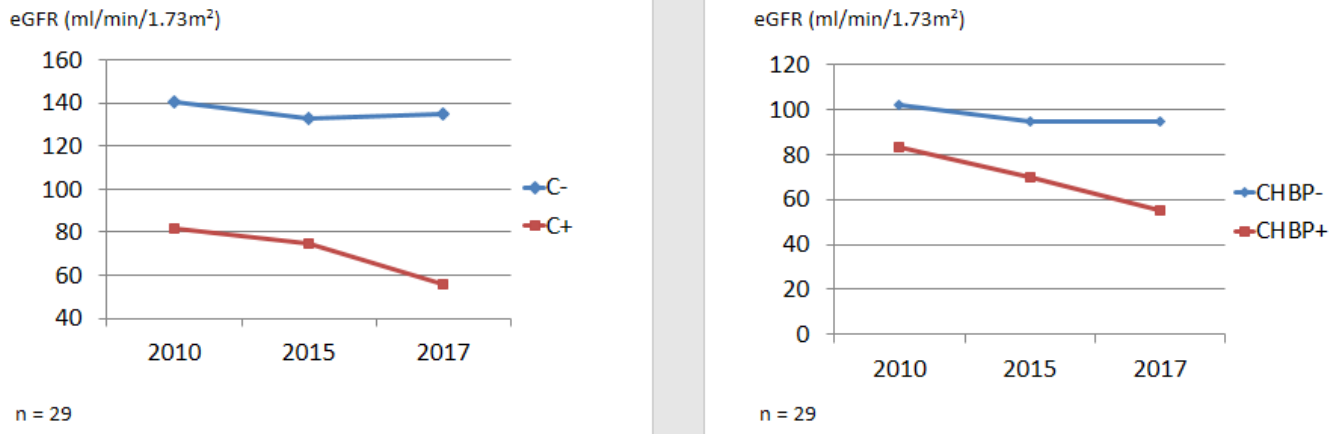


\section{Discussion:}

In this study, we aimed to correlate renal function with kidney complications and hypertension in ADPKD patients. As new advances in the treatment of ADPKD are now available, there is further need to rapidly identify those patients susceptible of suffering a rapid progress of the disease to establish treatment and adjust the follow-up. Our results suggest that patients who present at least one renal complication and/or hypertension have an increased deterioration of renal function. This difference is more notable if we compare patients with at least one renal complication and/or poorly controlled hypertension with those who do not present it.

To date, several factors have been related to the evolution of renal function in ADPKD patients, such as gene and type of mutation, hTKV, and hypertension. An additional -though less studiedfactor- is the development of clinical complications in these patients. These complications include hypertension, pain, macrohaematuria, cystic infection, pyelonephritis and nephrolithiasis. In some scores of risk progression, such as PROPKD, kidney complications and HBP are already considered but only in younger patients ( $<35$ years old) [13-14], and to the best of our knowledge there are very few studies in the literature related to ADPKD complication and disease progression $[1,2,12]$.

In this sense, there are no reviews assessing the frequency of all kidney complications. Separately, it has been seen that the frequency of macrohaematuria is $35-50 \%$ [20], whilst the frequency of nephrolithiasis is more than $25 \%$ [21] and the frequency of urinary tract infections is $30-50 \%$ [22]. Our study reviews the presence of complications and the evolution of renal function in patients with ADPKD Our patients showed a frequency of kidney complications of $72.4 \%$ which increased to $86.2 \%$ when HBP was included. The most common kidney complications detected were pain and cyst infection.

Hypertension occurs in $60 \%$ of patients before the decline of the renal function [4]. HBP is one of the most studied complications and is known to be related to a faster progression and to cardiovascular disease, the most common cause of mortality in these patients. Patients with high blood pressure show larger kidney volumes than those with normotension [23-24], and this is related to worse kidney function. Considering this previous knowledge, we initially divided patients 
into those with at least one complication and/or HBP $\left(\mathrm{C}_{+}\right)$and those without complication nor HBP (C-), but age appeared as a statistically significant factor. The age in group C-patients was 20 years younger than the $C_{+}$group. This is probably the main reason to explain the eGFR in $C_{+}$ group is higher than in the C- group. Yet, it is important to remark that the decrease in eGFR in $\mathrm{C}+$ group is greater than the decrease in eGFR in C- group, reaching a 25\% reduction of the filtration rate in the last two years of follow-up. This may suggest that kidney complications and hypertension could lead to a worse prognostic over the years.

It is widely known that bad controlled hypertension has worse effect in kidney function and other organ damage than well controlled hypertension. For this reason, we reanalyzed the data considering in the group of complications those patients that at least suffered one kidney complication and/or poorly controlled HBP $(\mathrm{CHBP}+)$, and patients without kidney complication and normotensive or controlled HBP (CHBP-). We found that these patients with complications and/or badly controlled HBP had worse kidney function compared with the other group. Importantly, the age factor is not significant in this new patient division, but the differences in eGFR decrease remain, thus suggesting that differences in kidney evolution are rather due to the complication, no to the age.

Based on these data, we can affirm that the clinical complications of ADPKD lead to a faster deterioration of renal function and progression to end-stage renal disease, especially in patients with uncontrolled hypertension. For this reason, we consider that "complications" including unoptimal control of HBP is a factor to take into account when identifying patients with faster progression in order to adjust the monitoring and assess the need to initiate a specific treatment, and highlight the importance of good blood pressure control. Yet, this is a cross-sectional observational study, and therefore can only describe associations but not causality relationships, and the number of patients included is limited. Also, this is a retrospective study, so it has not been possible to obtain information of genetic studies (they were only performed in certain cases) or hTKV (not standardized technique in our centre at the starting time of data collection). Despite not having this information, we still found differences in renal evolution. For this reason, we consider this study to be a good first approximation to highlight the importance of complications 
in the progression and prognostic of ADPKD. Although some prognostic scores, such as PROPKD, take into account kidney complications, in general, it is a poorly evaluated subject in the follow-up of the patients.

Both the PROPKD and the Mayo Model require complex complementary tests that are not routinely performed in many centers, such as MRI or genetic testing. Our proposal is a more orientative model but one that is based on the usual medical history in routine visits at doctor office, so it does not require more time or budget for imaging or genetic tests. Furthermore, in centers where the Mayo Model or PROPKD is used, it can provide even more information that these two models do not consider, and therefore provides a more complete overview of pathology and progression, without complicating routine clinical practice. Despite being a first approximation and not yet giving a numeral score such as the PROPKD or a complete classification such as the Mayo model, we think that it provides valuable information on factors that must be evaluated when creating a useful prognostic Score and we think that this is an important first step in this direction.

\section{Conclusions:}

In this study we found the following conclusions:

- ADPKD complications are very frequent: $72.4 \%$ of our patients present kidney complications of $72.4 \%$ and $86.2 \%$ present kidney complications and/or HBP.

- Patients with at least one complication and/or HBP have worse evolution in kidney function among years compared with those patients without any complication.

- Patients with at least one complication and/or poorly controlled HBP have even worse kidney function compared with those patients without complications or with controlled HBP.

- Complications of ADPKD cause a faster progression of renal disease, especially in patients with uncontrolled HBP. 


\section{List of abbreviations:}

ADPKD Autosomal dominant polycystic kindey disease

CKD Chronic kidney disease

eGFR Estimation of glomerular function rate

EMA European Medicines Agency

ESRD End-stage renal disease

HBP High blood pressure

HDL High density lipoprotein

hTKV Height adjusted total kidney volume

RRT Renal replacement therapy 


\section{Declarations:}

Ethics approval and consent: All patients provided informed consent and the protocol was conducted following the Helsinki declaration.

Consent for publication: not applicable.

Availability of data and materials: the datasets used and analyzed during this study are included in this published article.

Competing interests: The authors declare no conflicts of interest.

Funding: ISCIII-REDinREN (RD16/0009 Feder Funds).

\section{Authors' contributions:}

MPM: conception and design, acquisition of data, analysis and interpretation of data.

LCP: conception and design.

FEB: conception and design, analysis and interpretation of data.

JB: conception and design, analysis and interpretation of data.

MT: conception and design, analysis and interpretation of data.

All authors read and approved the final manustript.

Acknowledgments: not applicable. 


\section{Bibliography}

1. Grantham JJ, Mulamalla S, Swenson-Fields KI. Why kidneys fail in autosomal dominant polycystic kidney disease. Nat Rev Nephrol 2011; 7: 556-566

2. Sallee M, Rafat C, Zahar JR et al. Cyst infections in patients with autosomal dominant polycystic kidney disease. Clin J Am Soc Nephrol 2009; 4: 1183-1189

3. Suwabe $\mathrm{T}$, Araoka $\mathrm{H}$, Ubara $\mathrm{Y}$ et al. Cyst infection in autosomal dominant polycystic kidney disease: causative microorganisms and susceptibility to lipid-soluble antibiotics. Eur J Clin Microbiol Infect Dis 2015; 34: 1369-1379

4. Ecder T and Schrier R. Hypertension in autosomal-dominant polycystic kidney disease: early occurrence and unique aspects. JASN 2001 Jan;12(1):194-200

5. Orskov B, Romming S, V, Feldt-Rasmussen B, Strandgaard S. Improved prognosis in patients with autosomal dominant polycystic kidney disease in Denmark. Clin J Am Soc Nephrol 2010; 5: 2034-2039.

6. Perrone R, Muoksassi M, K. Romero et al. Total kdiney volumen is a prognostic biomarker of renal function decline and progression to end-stage renal disease in patients with autosomal dominant polycystic kidney disease. Kidney International reports (2017) 2, 442-450.

7. Soroka S, Alam, A, Bevilaccqua $M$ et al. Assessing risk of disease progression and pharmacological management of autosomal dominant polycystic kidney disease: a Canadian expert consensus. Canadian journal of Kidney Health and Disease 2017;4:112.

8. Cornec-Le Gall E, Audrézet MP, Chen JM, et al. Type of PKD1 mutation influences renal outcome in ADPKD. J Am Soc Nephrol. 2013;24(6):1006-1013. 
9. Porath B, Gainullin VG, Cornec-Le Gall E et al. Mutations in GANAB, encoding the glucosidase lla subunit, cause autosomal-dominant polycycistic kidney and liver disease. Am J Hum Genet 2016; 98: 1193-1207.

10. Grantham JJ, Torres VE, Chapman AB, et al. Volume progression in polycystic kidney disease. N Engl J Med. 2006;354(20):2122-2130.

11. Tangri N, Hougen I, Alam A et al. Total kidney volume as a biomarker of disease progression in autosomal dominant kidney disease. Can J. kidney Health Dis 2017: Mar $2 ; 4$.

12. Torrres VE, Craham JJ, Chapman AB et al. Potentially modifiable factors affecting the progression of autosomal dominant polycystic kidney disease. Clin J Am Soc nephrol $2011 ; 6: 640-647$.

13. Cornec-Le Gall E, Audrezet MP, Rousseau A et al. The PROPKD score: a new algorithm to predict renal survival in autosomal dominant polycystic kidney disease. J Am Soc Nephrol 2016; 27: 942-951

14. Cornec-Le Gall E, Blais J, Irazabal M et al. Can we further enrich autosomal dominant polycystic kidney disease clinical trials for rapidly progressive patients? Application of the PROPKD scores in the TEMPO trial. NDT 2017: 1-8.

15. Chapman AB, Devuyst O, Eckardt KU et al. Autosomal-dominant polycystic kidney disease (ADPKD): executive summary from a Kidney Disease: Improving Global Outcomes (KDIGO) Controversies Conference. Kidney Int. 2015 Jul; 88(1):17-27.

16. L. Giradat-Rotar, J. Braun, M. Puhan et al. Temporal and geographical external validation study extension of the Mayo Clinic prediction model to predict eCGF in the younger population of swiss ADPKD patients. BMC Nephrol 2017; 18: 241.

17. Pei Y, Obaji J, Dupuis A et al. Unified criteria for ultrasonographic diagnosis of ADPKD. J Am Soc Nephrol 2009; 20; 205-2012. 
18. Ars E, Bernis C, Fraga G et al. Spanish guidelines for the management of autosomal dominant polycystic kidney disease. Nephrol Dial Transplant 2014; 29 (suppl 4): iv 95105,

19. Kelleher C, McFann K, Johnson A et al. Characteristics of hypertension in young adults with autosomal dominant polycystic kidney disease compared with the general U.S. Population. Am J Hypertens 2004;17:1029-34.

20. Gabow PA, Duley I, Johnson AM. Clinical profiles of groos hematuria in autosomal sominant polycystic kidney disease. Am J Kidney Dis 1992; 20:140.

21. Torres VE, Wilson DM, Hattery RR, Segura JW. Renal stone disease in autosomal dominant polycystic kidney disease. Am J. Kidney Dis 1993; 22:513.

22. Sallée M, Rafat C, Zahar JR et al. Cyst infections in patients with autosomal dominant polycystic kidney disease. Clin J Am Soc Nephrol. 2009;4(7):1183. Epub 2009 May 21.

23. Chapman AB, Stephniakowski K, Rahbari-Oskoui F: Hypertension in autosomal dominant polycycistic kidney disease. Adv Chronic Kidney Dis 2010; 17: 153-63.

24. Gabow PA, Chapman AB, Johnson AM et al. Renal structure and hypertension in autosomal dominant polycycistic kidney disease. Kidney Int 1990; 38; 1177-80. 
Figures

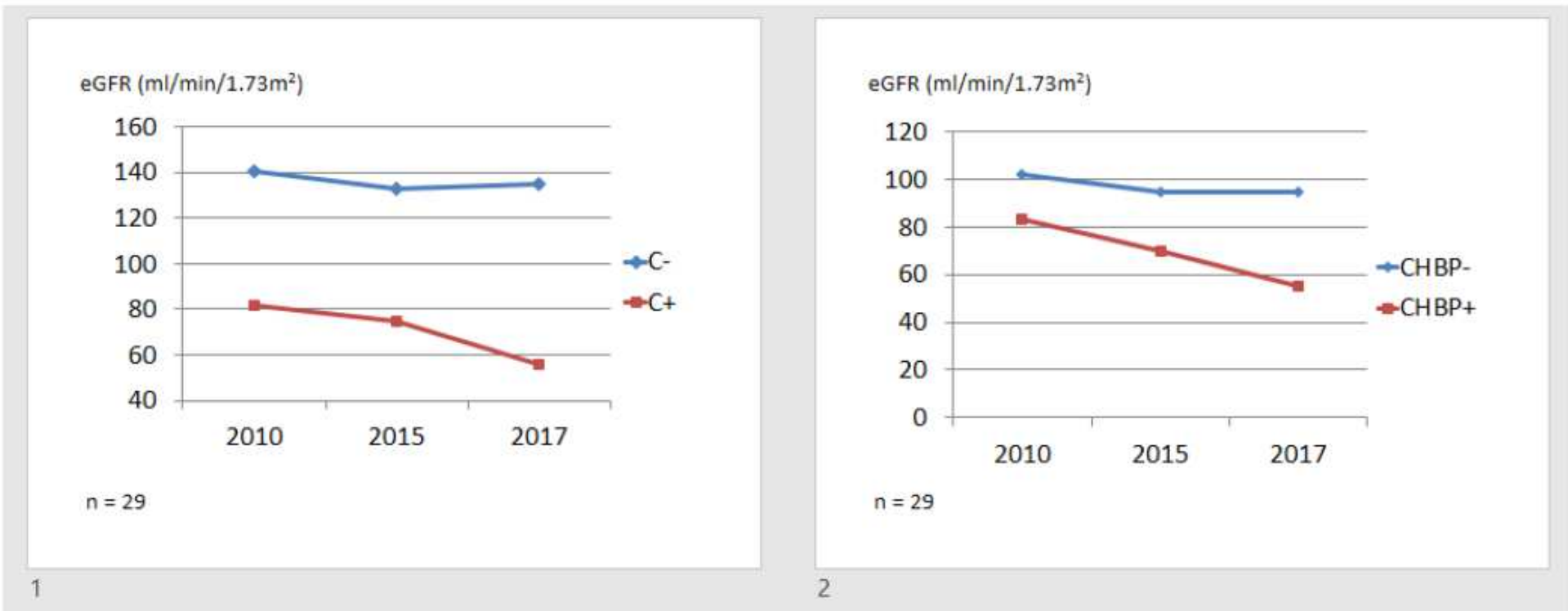

Figure 1

Evolution of decrease in eGFR in groups $\mathrm{C}$ - and $\mathrm{C}+(1)$ and $\mathrm{CHBP}$ - and $\mathrm{CHBP}+(2)$ : 